\title{
Effects of Isoperimetric Constraint for Reducing HSV-2 Infection Using Optimal Control Strategy
}

\author{
Samiha Islam Tanni, Jakia Sultana, Shamima Islam, Farzana Afroz and Md. Robiul Islam
}

\begin{abstract}
Optimal control is helpful for testing and comparing different vaccination strategies of a certain disease. Genital herpes is one of the most prevalent sexually-transmitted diseases globally. In this paper, we have proposed an optimal control problem applied to HSV-2 model after introducing the constraint and state variables. Optimal control problem is formulated based on ordinary differential equation and isoperimetric constraint in the vaccine supply is also included. Mathematical analysis such as the characterization of optimal control using Pontryagin's maximum principle is studied. Generally optimal control theory is used for finding the optimal way for implementing the strategies, minimizing the number of infectious and latent individuals and keeping the cost of implementation as low as possible. Here the optimality system is derived and solved numerically using a Runge-Kutta fourth order method and this is an iterative method. Using numerical simulation we observe that how the optimal vaccination schedule is altered by imposing isoperimetric constraint. Finally, on applying the isoperimetric constraint on the optimal control problem of HSV-2 epidemic model, we observe that optimal vaccination schedule with isoperimetric constraint indicates successful short-term control of the disease.
\end{abstract}

Index Terms-Herpes Simplex Virus Type-2 (HSV-2), Mathematical model, Optimal control, Characterization of the objective functional, Pontryagin's Maximunm Principle, Optimality system, Hamiltonian (H), Isoperimetric constraint

\section{INTRODUCTION}

$\mathrm{O}$ PTIMAL control theory is one of the important area of mathematics which has been popular in recent years. It is extensively used in controlling the spread of infectious diseases. It is a mathematical optimization method for deriving control policies. Lev Pontryagin and Richard Bellma [1] first worked on this theory. Optimal control deals with the problem of finding a control law for a given system such that a certain optimality criterion

DOI: https://doi.org/10.3329/gubjse.v7i0.54018

This paper was received on 18 May 2020, revised on 1 March 2021 and accepted on 19 April 2021.

Samiha Islam Tanni is with the Department of Computer Science and Engineering, Green University of Bangladesh, Dhaka, Bangladesh. E-mail: samiha@cse.green.edu.bd.

Jakia Sultana is with the Department of Computer Science and Engineering, Green University of Bangladesh, Dhaka, Bangladesh. E-mail: jakia@cse.green.edu.bd.

Shamima Islam is with the Department of Computer Science and Engineering, Green University of Bangladesh, Dhaka, Bangladesh. E-mail: shamima@cse.green.edu.bd.

Farzana Afroz is with the Department of Computer Science and Engineering, Green University of Bangladesh, Dhaka, Bangladesh. E-mail: farzana@cse.green.edu.bd.

Md. Robiul Islam is with the Department of Computer Science and Engineering, Green University of Bangladesh, Dhaka, Bangladesh. E-mail: robiul@ cse.green.edu.bd. is achieved. It is a powerful mathematical tool that can be used to make decisions involving complex biological situation [2]. It is often used in the control of the spread of most diseases for which either vaccine or treatment is available.

Herpes simplex virus type 2 (HSV-2), being a linear double-standed DNA virus of Herpesviridae family and Alphaherpesvirinae subfamily, is a sexually transmitted disease that inflicts severe public health burden globally. Its transmission is occurred by sexual contact or via the maternal-neonatal relationship. In recent times around the world at least 500 million people are infected by this virus [3]. The outbreak of HSV-2 virus is higher in the USA compared to Europe, Australia and New Zealand. Till now the awareness about the disease is not satisfactory and people may be transmitted by the virus during the periods of subclinical shedding.

In recent years, it is of great concern worldwide that many kinds of infectious diseases are spreading out. We need to prevent these diseases by taking effective measures and also take initiative to control the spreads of the infections. Both vaccination and treatment are useful for controlling the spread of infectious diseases but vaccination is assumed to be more efficient control strategy. We will design a mathematical modeling framework for gaining insights into the spread of HSV-2 in the body of an infected host, based on exists model [4]. We will also use optimal control theory with isoperimetric constraint to purpose effective strategies for controlling its spread. Since optimal control technique represents vaccination schedule easily for finite time period, so we choose this technique to gain insight into HSV-2 diseases dynamics. To implement the vaccination strategies, a small portion of current susceptible population is targeted for vaccination. Then the isoperimetric constraint is imposed in the optimal control problem and there are some basic elements for isoperimetric constraint: (i) the control $u(t)$ which arise in the objective functional and isoperimetric constraint, (ii) the state $Z(t)$, (iii) the state equation which is also called the isoperimetric constraint and it provides the dependence between the control and the state, (iv) the objective functional or cost functional which is extremized depending on the control and state. Our goal is to find the allowable control that extremizes the value of the objective 
functional by generating a satisfactory state. Then we exhibit how the optimal control with isoperimetric constraint is used to minimize the number of infected individuals and ultimately for preventing the disease.

\section{Formulation OF HS V-2 MATHEMATICAL MODEL}

Now we will design a HSV-2 transmission model (without any control). The basic model is designed by sub-dividing the total, homogeneously-mixed, heterosexual, sexuallyactive population at time $t$, denoted by $N(t)$, into mutually- exclusive compartments for individuals that are susceptible $S(t)$, exposed to HSV-2 without any clinical symptoms of the disease $E(t)$, infectious (virus shedding) with clinical symptoms of HSV-2 $H(t)$ and infectious but their infection is quiescent $Q(t)$, so that $N(t)=S(t)+E(t)+$ $H(t)+Q(t)$.

The increase of the susceptible population is occurred by the recruitment of new sexually-active individuals which are also assumed susceptible and the rate at which the susceptible population is increased is denoted by $b$ and this is the birth rate. This population is diminished by death at a rate $d$ and the acquisition of infection, following effective contact with infectious individuals (in the $H$ and $Q$ classes), at a rate $c$. The parameter $0 \leq \theta<1$ is called modification parameter and it indicates that the infectivity of the infectious individuals is lower in the quiescent class $Q$ compared to those in the $H$ class (that is, we assume that infectious individuals in the quiescent state are less infectious than nonquiescent infectious individuals, because we consider that the viral load is reduced in that stage). considering the above assumptions and parameters which are defined, we get the following equation which determine the rate of change of the susceptible population:

$$
\frac{d S}{d t}=b N(t)-d S(t)-c S(t)(H+\theta Q)(t) .
$$

Now we consider the case of exposed class. When the susceptible individuals are infected at the rate $\mathrm{c}$, then we get the population of exposed class which is reduced when the clinical symptoms by exposed individuals are developed at a rate $e$ and natural death rate $d$. Then we get,

$$
\frac{d E}{d t}=c S(t)(H+\theta Q)(t)-(e+d) E(t) .
$$

The population of infectious but non-quiescent individuals $H(t)$ is increased at the rates $e$ and $r$ which are the development of symptoms of exposed individuals and the reactivation of symptoms by individuals in the quiescent state respectively. Further, this population is diminished at the rates $q, d$ and $a_{l}$ which are the acquisition of quiescence status, natural death and disease-induced death rate respectively. Thus,

$$
\frac{d H}{d t}=e E(t)+r Q(t)-q H(t)-d H(t)-a_{1} H(t) .
$$

Similarly, for the quiescent class $Q(t)$, the rate of change of the population of individuals is increased at the rate $q$ which is the accession of quiescence individuals in the $\mathrm{H}(\mathrm{t})$ class and decreased at the rates $\mathrm{r}, \mathrm{d}$ and $\mathrm{a} 2$, which are re-activation of symptoms, natural death rate and disease-induced death rate respectively.

$$
\frac{d Q}{d t}=q H(t)-r Q(t)-d Q(t)-a_{2} Q(t) .
$$

The rate of change of the total population is given by

$$
\frac{d N}{d t}=(b-d) N(t)-\left(a_{1}+a_{2}\right)(H(t)+Q(t)) \text {. }
$$

The strategy of the control we adopt consists of a vaccination program. Our goal is to minimize the number of infected individuals. We introduce a control $u(t)$ into the basic model that represents the percentage of susceptible individuals being vaccinated at time $t$ which efforts as controls to curtail the spread of HSV-2. Here as a control class, piecewise continuous functions defined on $\left[0, t_{f}\right]$, with the bound $0 \leq u(t) \leq 1$ is chosen. It is to be noted that, $u(t)=1$ corresponds to maximal use of vaccination. The vaccination is absent for $\mathrm{u}(\mathrm{t})=0$.

So the controlled mathematical system is given by the following system of differential equations:

$$
\begin{aligned}
\frac{d S}{d t} & =b N(t)-d S(t)-c S(t)(H+\theta Q)(t)-u(t) S(t), \\
\frac{d E}{d t} & =c S(t)(H+\theta Q)(t)-(e+d) E(t)+\theta u(t) S(t), \\
\frac{d H}{d t} & =e E(t)+r Q(t)-q H(t)-d H(t)-a_{1} H(t)+ \\
& (1-\theta) u(t) S(t), \\
\frac{d Q}{d t}= & q H(t)-r Q(t)-d Q(t)-a_{2} Q(t), \\
\frac{d N}{d t}= & (b-d) N(t)-\left(a_{1}+a_{2}\right)(H(t)+Q(t)) .
\end{aligned}
$$

with initial conditions,

$S(0)=S_{0} \geq 0, E(0)=E_{0} \geq 0, H(0)=H_{0} \geq 0$,

$$
Q(0)=Q_{0} \geq 0, N(0)=N_{0} \text {. }
$$

A schematic diagram of the disease transmission among the individuals for the above model with vaccination control is shown in Fig. (1):

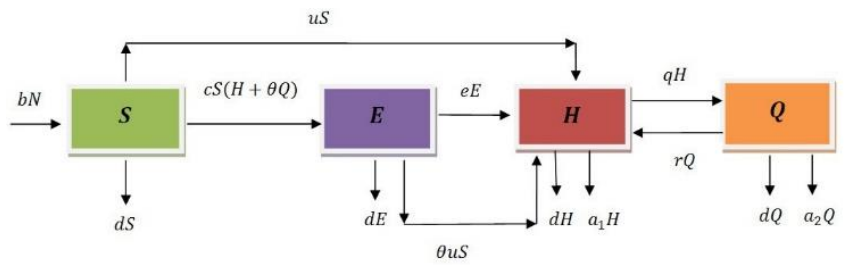

Fig. 1. HSV-2 model with vaccination

\section{Optimal CONTROL STRATEGy APPliEd to THE HSV-2 MODEL}

A. Formulation of our Optimal Control Problem We define our objective functional as

$$
J(u)=\int_{0}^{t_{f}}\left(A(H+\theta Q)(t)+u(t)^{2}\right) d t
$$


subject to the system of equation (1) while the control set $U$ is Lebesgue measurable and is defined as

$$
\begin{aligned}
& U=\{u(t) \text { is piecewise continuous function | } \\
& \left.\qquad 0 \leq u(t) \leq 1, \operatorname{in}\left[0, t_{f}\right]\right\} .
\end{aligned}
$$

Our aim is to minimize the total number of infected individuals as well as minimize the cost of vaccination given the initial population sizes of all four classes $S(0), E(0), H(0)$ and $Q(0)$. A is a weight parameter and this parameter indicates the comparative importance of the two terms in the functional. The first term in the objective functional, $A(H+\theta Q)(t)$ stands for the total number of individuals who are infected and is taken as a measure of the death associated with the outbreak. We also consider a quadratic cost on the control, which is the simplest and widest used nonlinear representation of vaccination cost [5].

\section{Characterization OF AN OPTIMAL CONTROL}

Now we have to characterize the optimal control. For this we apply Pontryagin's maximum principle and the Hamiltonian $\mathrm{H}$ is also defined. For the optimal control $u^{*}$, we get a solution of the corresponding state system (1) which is $X^{*}(t)=$ $\left(S^{*}(t), E^{*}(t), H^{*}(t), Q^{*}(t), N^{*}(t)\right)$. We see the existence of the adjoint variables $\lambda_{S}(t), \lambda_{E}(t), \lambda_{H}(t), \lambda_{Q}(t), \lambda_{N}(t)$ and these variables satisfy the following equations:

$$
\begin{aligned}
& \lambda_{S}^{\prime}=\lambda_{S}(d+c(H+\theta Q)+u)-\lambda_{E}(c(H+\theta Q)+\theta u)-\lambda_{H}((1-\theta) u, \\
& \lambda_{E}^{\prime}=\lambda_{E}(e+d)-\lambda_{H} e, \\
& \lambda_{H}^{\prime}=-A+\left(\lambda_{S}-\lambda_{E}\right) c S+\lambda_{H}\left(q+a_{1}+d\right)-\lambda_{Q q}+\lambda_{N}\left(a_{1}+a_{2}\right), \\
& \lambda_{Q}^{\prime}=-A \theta+\left(\lambda_{S}-\lambda_{E}\right) c S \theta-\lambda_{H} r+\lambda_{Q}\left(r+a_{2}+d\right)+\lambda_{N}\left(a_{1}+a_{2}\right), \\
& \lambda_{N}^{\prime}=\lambda_{N}(d-b)-\lambda_{S} b .
\end{aligned}
$$

with transversality conditions

$$
\lambda_{i}\left(t_{f}\right)=0, i=S, E, H, Q, N .
$$

Furthermore, the optimal control $u^{*}(t)$ is given by

$$
u^{*}=\min \left[1, \max \left[0, \frac{S\left(\lambda_{S}-\lambda H\right)-\theta S\left(\lambda_{E}-\lambda_{H}\right)}{2}\right]\right] .
$$

Proof We have to find the optimal vaccination schedule and for this we use Pontryagin's Maximum Principle [6]. The Hamiltonian is defined as

$$
\begin{aligned}
H= & A(H+\theta Q)+u^{2}+\lambda_{S}(b N-d S-c S(H+\theta Q)-u S) \\
& +\lambda_{E}(c S(H+\theta Q)-(e+d) E+\theta u S)+\lambda_{H}(e E+r Q \\
& \left.-\left(q+a_{1}+d\right) H+(1-\theta) u S\right)+\lambda_{Q}\left(q H-\left(r+a_{2}+d\right) Q\right) \\
& +\lambda_{N}\left((b-d) N-\left(a_{1}+a_{2}\right)(H+Q)\right) .
\end{aligned}
$$

Here the associated adjoints for the state $S, E, H, Q, N$ are $\lambda_{S}, \lambda_{E}, \lambda_{H}, \lambda_{Q}, \lambda_{N}$ respectively. For finding the differential equation for the associated adjoin, the Hamiltonian is differentiated with respect to each state variable. Since the final time values of $S, E, H, Q$ and $N$ are not fixed, so the values of the associated adjoints at the final time are zero. Therefore the adjoint system becomes

$$
\begin{aligned}
& \lambda_{S}^{\prime}=\lambda_{S}(d+c(H+\theta Q)+u)-\lambda_{E}(c(H+\theta Q)+\theta u)-\lambda_{H}((1-\theta) u), \\
& \lambda_{E}^{\prime}=\lambda_{E}(e+d)-\lambda_{H} e, \\
& \lambda_{H}^{\prime}=-A+\left(\lambda_{S}-\lambda_{E}\right) c S+\lambda_{H}\left(q+a_{1}+d\right)-\lambda_{Q} q \\
& +\lambda_{N}\left(a_{1}+a_{2}\right) \text {, } \\
& \lambda_{Q}^{\prime}=-A \theta+\left(\lambda_{S}-\lambda_{E}\right) c S \theta-\lambda_{H} r+\lambda_{Q}\left(r+a_{2}+d\right) \\
& +\lambda_{N}\left(a_{1}+a_{2}\right) \text {, } \\
& \lambda_{N}^{\prime}=\lambda_{N}(d-b)-\lambda_{S} b .
\end{aligned}
$$

The transversality conditions for this system is

$$
\lambda_{S}\left(t_{f}\right)=0, \lambda_{E}\left(t_{f}\right)=0, \lambda_{H}\left(t_{f}\right)=0, \lambda_{Q}\left(t_{f}\right)=0, \lambda_{N}\left(t_{f}\right)=0
$$

By optimal condition we have $\left.\frac{\partial H}{\partial u}\right|_{u=u^{*}}=0$

$$
\begin{aligned}
& \Rightarrow 2 u^{*}-\lambda_{S} S+\lambda_{E} \theta S+(1-\theta) \lambda_{H} S=0 \\
& \Rightarrow 2 u^{*}=\lambda_{S} S-\lambda_{E} \theta S-(1-\theta) \lambda_{H} S \\
& \Rightarrow 2 u^{*}=\lambda_{S} S-\lambda_{E} \theta S-\lambda_{H} S+\theta \lambda_{H} S \\
& \Rightarrow 2 u^{*}=S\left(\lambda_{S}-\lambda_{H}\right)-\theta S\left(\lambda_{E}-\lambda_{H}\right) \\
& \Rightarrow u^{*}=\frac{S\left(\lambda_{S}-\lambda_{H}\right)-\theta S\left(\lambda_{E}-\lambda_{H}\right)}{2}
\end{aligned}
$$

Since $u^{*}$ denotes the optimal control, we now solve for $u^{*}$ and obtain the following control set:

$$
u^{*}=\frac{S\left(\lambda_{S}-\lambda_{H}\right)-\theta S\left(\lambda_{E}-\lambda_{H}\right)}{2} .
$$

By standard control agreement involving bounds on the controls, we conclude

$$
u^{*}= \begin{cases}0 & \text { if } \frac{S\left(\lambda_{S}-\lambda_{H}\right)-\theta S\left(\lambda_{E}-\lambda_{H}\right)}{2}<0, \\ 1 & \text { if } 0 \leq \frac{S\left(\lambda_{S}-\lambda_{H}\right)-\theta S\left(\lambda_{E}-\lambda_{H}\right)}{2} \leq 1, \\ 2 & \text { if } \frac{S\left(\lambda_{S}-\lambda_{H}\right)-\theta S\left(\lambda_{E}-\lambda_{H}\right)}{2}>1 .\end{cases}
$$

In compact notion we get,

$$
u^{*}=\min \left[1, \max \left[0, \frac{S\left(\lambda_{S}-\lambda_{H}\right)-\theta S\left(\lambda_{E}-\lambda_{H}\right)}{2}\right]\right] .
$$

The state and the adjoint system both constitute the optimality system and the initial and transversality conditions together with the characterization of the optimal control are also included. Now we can utilize the characterization of the optimal 
control to get the following optimality system:

$$
\begin{aligned}
& S^{*}=b N^{*}-d S^{*}-c S^{*}\left(H^{*}+\theta Q^{*}\right)-u^{*} S^{*}, \\
& E^{*}=c S^{*}\left(H^{*}+\theta Q^{*}\right)-(e+d) E^{*}+\theta u^{*} S^{*}, \\
& H^{*}=e E^{*}+r Q^{*}-\left(q+a_{1}+d\right) H^{*}+(1-\theta) u^{*} S^{*} \text {, } \\
& Q^{*}=q H^{*}-\left(r+a_{2}+d\right) Q^{*}, \\
& N^{*}=(b-d) N^{*}-\left(a_{1}+a_{2}\right)\left(H^{*}+Q^{*}\right) \text {, } \\
& \lambda_{S}^{\prime}=\lambda_{S}\left(d+c\left(H^{*}+\theta Q^{*}\right)+u\right) \\
& -\lambda_{E}\left(c\left(H^{*}+\theta Q^{*}\right)+\theta u^{*}\right)-\lambda_{H}\left((1-\theta) u^{*}\right), \\
& \lambda_{E}^{\prime}=\lambda_{E}(e+d)-\lambda_{H} e \text {, } \\
& \lambda_{H}^{\prime}=-A+\left(\lambda_{S}-\lambda_{E}\right) c S^{*}+\lambda_{H}\left(q+a_{1}+d\right)-\lambda_{Q} q \\
& +\lambda_{N}\left(a_{1}+a_{2}\right) \\
& \lambda_{Q}^{\prime}=-A \theta+\left(\lambda_{S}-\lambda_{E}\right) c S^{*} \theta-\lambda_{H} r+\lambda_{Q}\left(r+a_{2}+d\right) \\
& +\lambda_{N}\left(a_{1}+a_{2}\right) \\
& \lambda_{N}^{\prime}=\lambda_{N}(d-b)-\lambda_{S} b \text {. }
\end{aligned}
$$

\section{HSV-2 MODEL WITH ISOPERIMETRIC CONSTRAINT}

Now we want to restrict our vaccination supply. We consider the case which implies the limited number of vaccination supplies and find the optimal vaccination schedule where only a given number of people are vaccinated.

The extension of our model is occured to get the idea about the practical situation in which the supply of vaccine is limited. If $Y$ denotes the total number of susceptible people we can vaccinate over $t_{f}$ years, then we get the following integral:

$$
\int_{0}^{t_{f}} S(t) u(t) d t=Y
$$

Here we get an integral constraint and this types of constraints are called isoperimetric constraints [2]. Now we introduce another state variable, $Z(t)$ to handle this isoperimetric constraint. Then we get

$$
\begin{gathered}
Z^{\prime}(t)=S(t) u(t) \\
Z(0)=0 \\
Z\left(t_{f}\right)=Y
\end{gathered}
$$

The term $Z\left(t_{f}\right)=Y$ indicates that all the vaccines should be used. So it will be more realistic if we work with $Z\left(t_{f}\right) \leq Y$.

From the numerical point of view this change is completely harmless. Considering all these we form a new optimality system which will be helpful for minimizing total number of infectious and quiescent infectious individuals and also the overall cost of the vaccination will be minimized over $t_{f}$ years. We can state the problem as follows:

$$
\min _{u}^{t_{f}} \int_{0}\left(A\left(H+\theta Q(t)+u(t)^{2}\right) d t\right.
$$

where the control set is

$U=\left\{u:\left[0, t_{f}\right] \rightarrow[0,1] \mid u\right.$ is piecewise continuous function $\}$.

Now we use Pontryagin's Maximum Principle [6] for finding the optimal vaccination schedule. At first we have to define the Hamiltonian as follows:

$$
\begin{aligned}
H= & A(H+\theta Q)+u^{2}+\lambda_{S}(b N-d S-c S(H+\theta Q)-u S) \\
& +\lambda_{E}(c S(H+\theta Q)-(e+d) E+\theta u S)+\lambda_{H}(e E+r Q \\
& \left.-\left(q+a_{1}+d\right) H+(1-\theta) u S\right)+\lambda_{Q}\left(q H-\left(r+a_{2}+d\right) Q\right) \\
& +\lambda_{N}\left((b-d) N-\left(a_{1}+a_{2}\right)(H+Q)\right)+\lambda_{Z}(S u) .
\end{aligned}
$$

Here we get the associated adjoints $\lambda_{S}, \lambda_{E}, \lambda_{H}, \lambda_{Q}, \lambda_{N}$ and $\lambda_{Z}$ for the state $S, E, H, Q, N$ and $Z$ respectively. Since this time we have added constrains to the Hamiltonian, so we have to differentiate the Hamiltonian with respect to each state variable to find the differential equation for the associated adjoint. We also consider the fact that since $S, E, H, Q$ and $N$ do not have fixed values at the final time, it is obvious that the values of the associated adjoints at the final time are zero. Since $Z$ has initial and terminal conditions, $\lambda_{Z}$ has no transversality condition. Therefore, the adjoint system (4) is extended to

$$
\begin{aligned}
& \lambda_{S}^{\prime}=\lambda_{S}(d+c(H+\theta Q)+u)-\lambda_{E}(c(H+\theta Q) \\
& +\theta u)-\lambda_{H}((1-\theta) u)-\lambda_{Z} u, \lambda_{S}\left(t_{f}\right)=0 \\
& \lambda_{E}^{\prime}=\lambda_{E}(e+d)-\lambda_{H} e, \lambda_{E}\left(t_{f}\right)=0 \\
& \lambda_{H}^{\prime}=-A+\left(\lambda_{S}-\lambda_{E}\right) c S+\lambda_{H}\left(q+a_{1}+d\right)-\lambda_{Q} q \\
& +\lambda_{N}\left(a_{1}+a_{2}\right), \lambda_{H}\left(t_{f}\right)=0 \\
& \lambda_{Q}^{\prime}=-A \theta+\left(\lambda_{S}-\lambda_{E}\right) c S \theta-\lambda_{H} r+\lambda_{Q}\left(r+a_{2}+d\right) \\
& +\lambda_{N}\left(a_{1}+a_{2}\right), \lambda_{Q}\left(t_{f}\right)=0 \\
& \lambda_{N}^{\prime}=\lambda_{N}(d-b)-\lambda_{S} b, \lambda_{N}\left(t_{f}\right)=0 \\
& \lambda_{Z}^{\prime}=0
\end{aligned}
$$

By optimal condition we have $\left.\frac{\partial H}{\partial u}\right|_{u=u^{*}}=0$

$\Rightarrow 2 u^{*}-\lambda_{S} S+\lambda_{E} \theta S+(1-\theta) \lambda_{H} S+\lambda_{Z} S=0$

$\Rightarrow 2 u^{*}=\lambda_{S} S-\lambda_{E} \theta S-(1-\theta) \lambda_{H} S-\lambda_{Z} S$

$\Rightarrow 2 u^{*}=\lambda_{S} S-\lambda_{E} \theta S-\lambda_{H} S+\theta \lambda_{H} S-\lambda_{Z} S$

$\Rightarrow 2 u^{*}=S\left(\lambda_{S}-\lambda_{H}-\lambda_{Z}\right)-\theta S\left(\lambda_{E}-\lambda_{H}\right)$

$\Rightarrow u^{*}=\frac{S\left(\lambda_{S}-\lambda_{H}-\lambda_{Z}\right)-\theta S\left(\lambda_{E}-\lambda_{H}\right)}{2}$

For the implementation of constraints, $u^{*}$ denotes the optimal control as earlier and we also solve for $u^{*}$ to get the following control sets:

$$
u^{*}=\frac{S\left(\lambda_{S}-\lambda_{H}-\lambda_{Z}\right)-\theta S\left(\lambda_{E}-\lambda_{H}\right)}{2}
$$

By standard control agreement involving bounds on the controls, we conclude

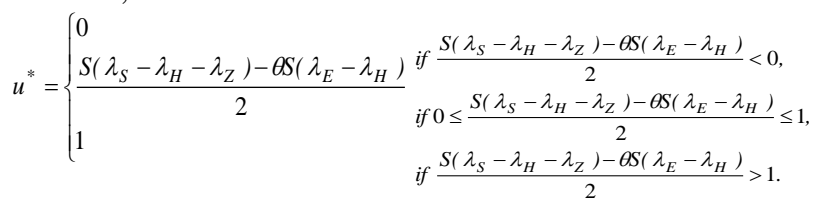

In compact notion (5) is also extended to

$u^{*}=\min \left[1, \max \left[0, \frac{S\left(\lambda_{S}-\lambda_{H}-\lambda_{Z}\right)-\theta S\left(\lambda_{E}-\lambda_{H}\right)}{2}\right]\right]$. 


\section{NUMERICAL IMPLEMENTATION FOR CONSTRAINED MODEL}

Now we solve the state and adjoint differential equations numerically and make a combination with the control characterization to illustrate our results. We have to compute the optimal state values where an initial guess for the control is given. The optimal state values can be computed by solving the equations (1), (6) with initial conditions $Z(0)=0$ and (2). Here the objective functional (3) is also used. In this case we use the Runge Kutta method of the fourth order and the equations are solved forward in time. After solving these equations we get the state values and the resulting state values are placed in adjoint equations (8). These adjoint equations with given final conditions are then solved backwards in time. For this case a fourth order Runge Kutta method is again employed. We use both state ad adjoint values to update the control using the characterization (9) and (10) which is our main focus. The entire process repeats itself and it terminates when current state, adjoint, and control values are sufficiently close to successive values. We can see the results for such iterative methods [7].

For the equation (8), we can write $\lambda_{Z}(t)=k$ for all $t \in\left[0, t_{f}\right]$ where $k$ is an unknown constant. The value $Z(t)$ directly affected by the value $\lambda_{Z}(t)=k$. We need to find the appropriate value of $\mathrm{k}$ and for this we use Matlab program. The optimal solutions satisfy both endpoint conditions, $Z(0)=0$ and $Z\left(t_{f}\right)=Y$ : At first we consider the case where $k=0$. We have already explained about optimal state, control, and adjoint values and in this case we compute these values. As we have mentioned earlier that, the state equations are solved forward in time and the condition $Z(0)=0$ is always satisfied. But we have to analyze the result for the condition $Z\left(t_{f}\right)$ because it is important to check whether the mentioned process is suitable for the condition $Z\left(t_{f}\right)$. After testing with all sets of parameters, we have found that larger $k$ values correspond to small values of $Z\left(t_{f}\right)$.

Now we analyze the changes in the the value $k$. Depending the value of $Z\left(t_{f}\right)$, it is increased or decreased associating with $k=$ 0 . Again it is necessary to compute the values of optimal state, adjoint, and control values. We obtain the value of $Z\left(t_{f}\right)$ which is associated with the inputted value of $Y$ and it changes the value of $\mathrm{k}$ accordingly. We can consider the current $\mathrm{k}$ value and its associated control, states and adjoints optimal if an only if $Z\left(t_{f}\right)=Y$ is reasonably satisfied. We will input different values of $\mathrm{k}$ for finding the optimal values of state, adjoint, and control values and after getting the correct $\mathrm{k}$ value, the process terminates [8].

For the simulation process we use some parameters which are described in the table 1 and the parameters are chosen arbitrarily. In this case, vaccination is simulated for 20 years.

TABLE I

DESCRIPTION AND PARAMETER VALUES OF THE HSV-2 MODEL

\begin{tabular}{|c|c|c|c|}
\hline ariable & Description & Initial Values & \\
\hline $\begin{array}{l}S_{0} \\
E_{0} \\
H_{0} \\
Q_{0}\end{array}$ & $\begin{array}{l}\text { initial suscrptible indwividuals } \\
\text { intial aposed indivivuals } \\
\text { initial infectious individuals } \\
\text { intitial quiescent individuals }\end{array}$ & $\begin{array}{l}1500 \\
300 \\
50 \\
50\end{array}$ & $\begin{array}{l}\begin{array}{l}\text { Assumned] } \\
\text { [Assumed] } \\
\text { [Assumed] } \\
\text { [Assumed] }\end{array} \\
\end{array}$ \\
\hline Parameters & Definition of Paramerers & Tritial values & \\
\hline $\begin{array}{l}b \\
d \\
c \\
e \\
r \\
q \\
a_{1, a} \\
\theta \\
A \\
t_{f}\end{array}$ & 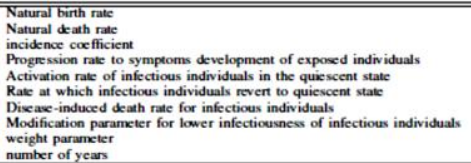 & $\begin{array}{l}0.725 \\
0.7 \\
.001 \\
0.6 \\
0.4 \\
0.5 \\
0.1,0.09 \\
0.5 \\
0.1 \\
20\end{array}$ & $\begin{array}{l}\text { Assumed] } \\
{[\text { Assumed] }} \\
{[91} \\
{[\text { Asumed] }} \\
{[\text { Assumed] }} \\
{[\text { Assumed] }} \\
{[\text { Assumed] }} \\
{[4]} \\
{[10]}\end{array}$ \\
\hline
\end{tabular}

\section{A. Effects of Isoperimetric Constraint}

Now we analyze the effcts of isoperimetric constraint. For this it is necessary to look at the optimal vaccination schedule given in the objective functional of (7) where there is no constraint on the number of susceptible population. To understand the effect when the isoperimetric constraint is absent, we remove the state variable $Z(t)$ from the optimal control problem. After removing the state variable we get a new optimality system and this system is solved using Matlab program.

Now we use the values in Table 1 to run the new program and we get the optimal vaccination schedule and corresponding population dynamics.
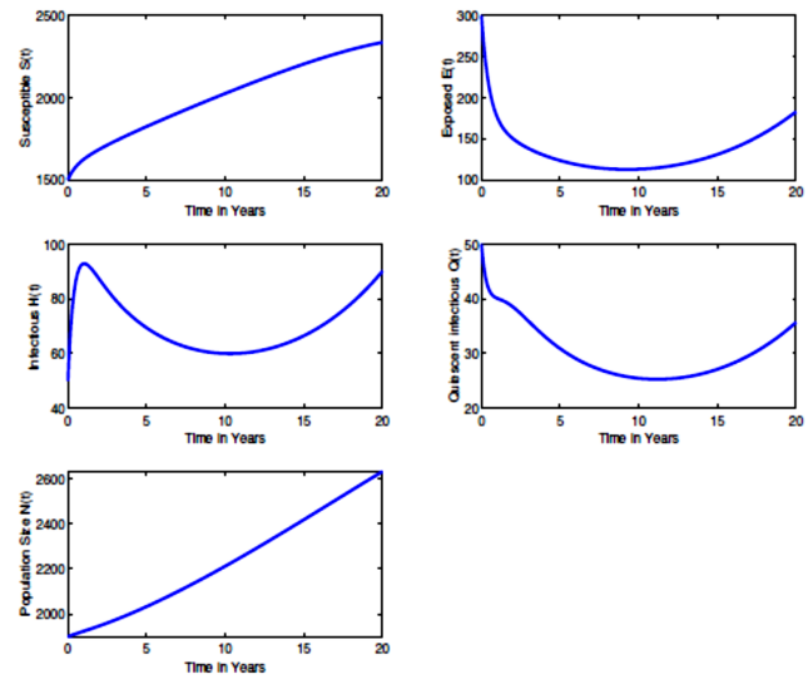

Fig. 2. HSV-2 model without isoperimetric constraint, parameter values are taken from Table 1, with $k=0$

Unlimited supply of vaccines: In Fig. 2, first we consider the case where $Y=+\infty$, i.e, we treat the case where unlimited number of vaccines is available over the period of time, which means that we can vaccinate the susceptible individuals as many as we choose during the 20 years.
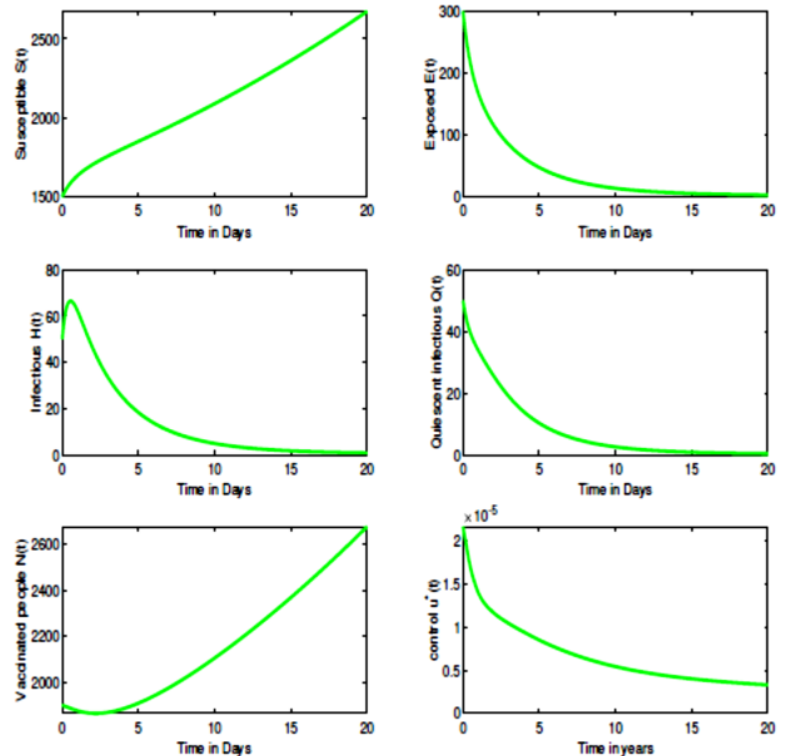

Fig. 3. HSV-2 model with isoperimetric constraint, parameter values are taken from Table 1 , here $Y=3000$ and $k .=: 000985223$ 
Overall limited supply of vaccines: Now we consider the case where $Y=3000$. This means we do have a limited number of 3000 vaccines to be used during the 20 years interval. Note that here we impose the end state $Z(20)$ to be less or equal to 3000 . Thus we allow the possibility of having no used vaccines in the end of 20 years. In this case we run the original program with $Z=3000$. The program terminates at 0:00098522748905508 corresponding to $Z\left(t_{f}\right)=3000$. The optimal vaccination schedule and corresponding population dynamics are displayed in Fig. 3.
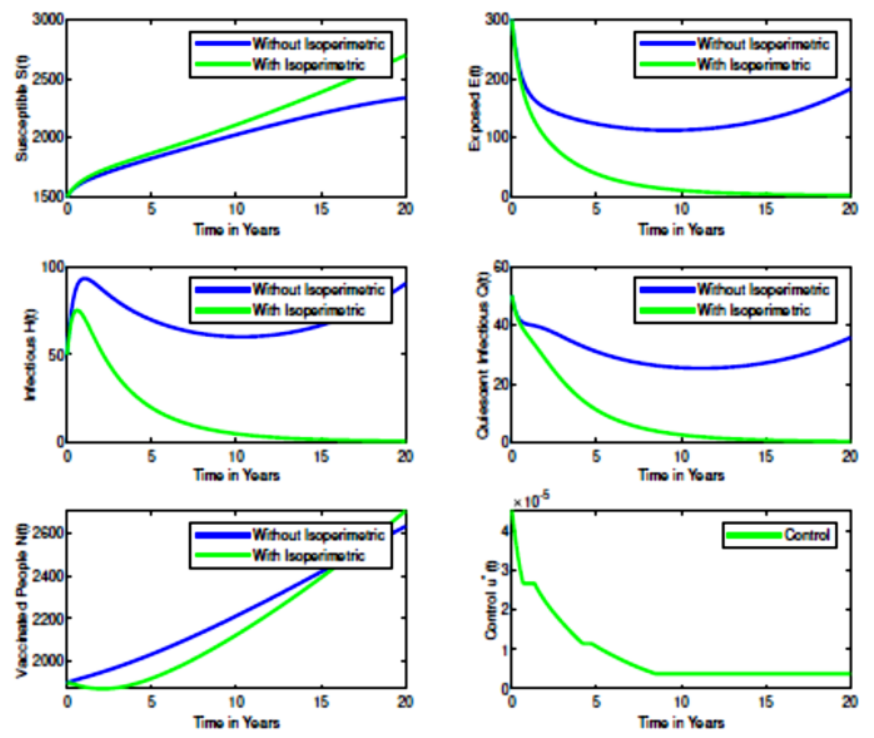

Fig. 4. HSV-2 model with and without isoperimetric constraint, parameter values are taken from Table 1, with $k .=0$ and $k=0: 000985223$

Comparison: In Fig. 4, comparison between Fig. 2 and Fig. 3 is observed. Comparing the schedule we observe that there is a mentionable change in the infection and control variable after implementing isoperimetric constraint. From the figure with and without isoperimetric constraint we observe that isoperimetric constraint can play an important role for reducing the infection. In both cases, the infectious and quiescent infectious population decrease to zero. Thus we see that the model with isoperimetric constraint have positive effect for controlling HSV-2 infection during the 20-year time period.

\section{CONCLUSION}

We have proposed an extended HSV-2 model subject to isoperimetric constraint to study the case where the vaccination process is bounded at each instant $t$. The isoperimetric constraint is enforced during the whole process of a vaccination program. Here we have studied the situation with and without isoperimetric constraint and the effects of added constraint. Numerical results illustrate the optimal vaccination schedule with and without the constraint. Simulation results also indicate that the proposed control strategy is effective for reducing the number of infected individuals and the cost of vaccination because after implementing the isoperimetric constraints the population of the infectious and quiescent infectious classes have reduced significantly. The optimal control strategy with isoperimetric constraint also suggests that what amount of limited vaccination should be given to the patients to reduce the viral infection to improve physical condition of the patient. So we can say optimal vaccination schedule with isoperimetric constraint gives significant control over disease for a finite time interval.

\section{ACKNOWLEDGEMENTS}

This work was supported in part by the Center for Research, Innovation, and Transformation (CRIT) of Green University of Bangladesh (GUB).

\section{REFERENCES}

[1] Sethi, S. P. and G. L. Thompson. Optimal Control Theory: Applications to Management Science and Economics, Kluwer, Boston, 2nd edition, 2000.

[2] S. Lenhart, J. Wortman. Optimal control applied to biological models. Taylor and Francis, Boca Raton, (2007).

[3] Looker KJ, Garnett GP, Schmid GP. An estimate of the global prevalence and incidence of herpes simplex virus type 2 infection. Bull World Health Organ. vol. 86(10)(2008):805-812.

[4] C.N. Podder and A. B.Gumel. Qualitative dynamics of a vaccination model for HSV-2. IMA Journal of Applied Mathematics. vol. 75 (1)(2010): 75-107.

[5] Y. Zhou, H. Liu. Stability of Periodic Solutions for an SIS Model with Pulse Vaccination. Mathematical and Computer Modelling. vol. 38 (2003): 299-308.

[6] Pontryagin, L. S., V. G. Boltyanskii, R. V. Gamkrelize, and E. F. Mishchenko. The Mathematical Theory of Optimal Processes, New York, Wiley, 1962.

[7] Hackbusch, W. K. A Numerical Method for Solving Parabolic Equations with Opposite Orientations, Computing. vol. 20(1978): 229-240.

[8] E. A Bakare, Yongzheng Sun. On the Optimal Control of Vaccination and treatments for an SIR-Epidemic with infected immigrants. Applied Mathematical Sciences, (2014), Hikari Ltd.

[9] G. Zaman, Y. Kang, I. Jung. Stability analysis and optimal vaccination of an sir epidemic model. BioSystems. vol. 93(2008): 240-249.

[10] W. H. Fleming and R. W. Rishel. Deterministic and Stochastic Optimal Control. Springer-Verlag, 1975.

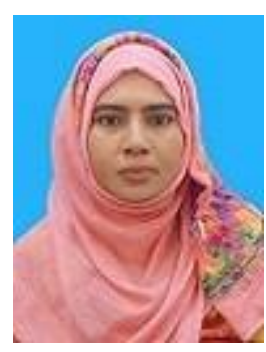

Samiha Islam Tanni was Born in Manikganj district in 1989. She received her BS degree in Mathematics and MS degree in Applied Mathematics in 2013 and 2015, respectively from University of Dhaka (DU). Currently she is serving as a lecturer (Mathematics) in the department of Computer Science and Engineering (CSE) in the Green University of Bangladesh (GUB) since September, 2018. She was also a field researcher of a project named Simon project which was organised by the primak group. Her research interests include Mathematical Modeling in Biology, Optimal Control Technique for reducing infectious diseases, Dynamical System etc. 


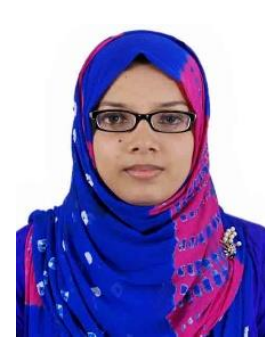

Jakia Sultana was born in Tangail district. She obtained her B.S. (Hons.) degree in Mathematics and M.S. degree in Applied Mathematics from Department of Mathematics, University of Dhaka in 2013 and 2015 respectively. She worked as a lecturer in Sonargaon University from 4th May, 2015 to 15th January, 2016. She joined Green University of Bangladesh as a lecturer on 16th January, 2016. Currently she is serving as a Senior Lecturer (Mathematics) in the department of Computer Science and Engineering (CSE) in the Green University of Bangladesh (GUB). Her research interests include mathematical modeling in Biology, mathematical fluid dynamics, Optimal control technique, dynamical systems, Operations Research and Optimization.

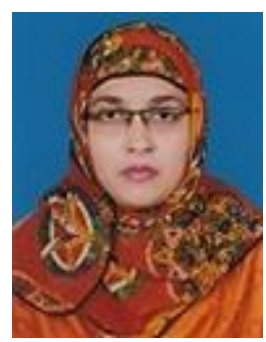

Shamima Islam was born in Chandpur district in 1987. She received her BS degree in Mathematics and MS degree in Applied Mathematic in 2011 and 2013 respectively from University of Dhaka (DU). Currently she is serving as a Senior Lecturer (Mathematics) in the department of Computer Science and Engineering (CSE) in the Green University of Bangladesh (GUB) since 05 September, 2014. Her research interests include mathematical modeling and simulation of fluidic phenomena, mathematical fluid dynamics.

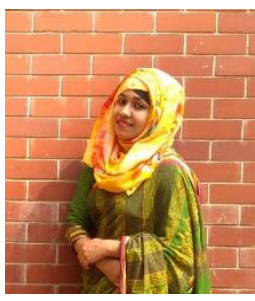

Farzana Afroz was born in Lakshmipur district in 1993. She received her BS degree in Mathematics and MS degree in Applied Mathematics in 2017 and 2019 respectively from University of Dhaka (DU). Currently she is serving as a Lecturer (Mathematics) in the department of Computer Science and Engineering (CSE) in Green University of Bangladesh (GUB) since 18 September, 2019. Her research interest includes financial mathematics and stochastic modeling, financial risk management, actuarial science.

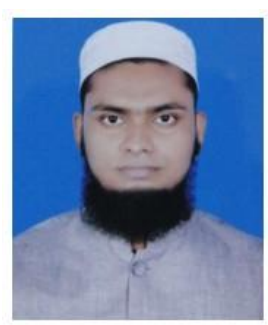

Md. Robiul Islam was born in Chapainawabganj district in 1994. He received his B.S (Hons) degree in Mathematics and M.S degree in Applied Mathematics in 2015 and 2016 respectively from University of Dhaka (DU). Currently he is serving as a lecturer (Mathematics) in the department of Computer Science and

Engineering (CSE) in the Green University of Bangladesh (GUB) since September, 2018. His research interests includes Modeling and Simulation of Fluidic Phenomena, Dusty Fluid Model, Application of Linear Algebra 